\title{
Zhou Method for the Solutions of System of Proportional Delay Differential Equations
}

\author{
S.O. Edeki ${ }^{{ }^{*}}$, and G.O. Akinlabi ${ }^{1}$ \\ ${ }^{1}$ Department of Mathematics, Covenant University, Ota, Zip Code: 112212, Nigeria
}

\begin{abstract}
In this paper, we consider a viable semi-analytical approach for the approximate-analytical solutions of certain system of functional differential equations (SFDEs) engendered by proportional delays. The proposed semi-analytical technique is built on the basis of the classical Differential Transform Method (DTM). The effectiveness and robustness of the proposed technique is illustratively demonstrated and the results are compared with their exact forms. We note also that using this method, the SFDEs with proportional delays need not be converted to SFDEs with constant delays before obtaining their solutions, and no symbolic calculation or initial guesstimates are required.
\end{abstract}

\section{Introduction}

Delay Differential Equation (DDE) is a functional differential equation (FDE) which arises when the rate of change of a time-dependent process is not only determined by its present state but also by a certain previous state in its mathematical modeling. That is, the rate of change of an unknown function depends not just on the values of the unknown function and its independent variable but also on the time values less than the independent variable [1].

This class of differential problems is encountered in many models of real life occurrences and has a wide range of applications in science and engineering. For instance, many of the processes in biology, engineering, physics, medicine, chemistry, and so on involve time delays. Different studies have been conducted by different authors on DDEs. We mention some of these as follows: Houwen and Sommeijer [2] dealt with the stability properties of Linear Multistep Methods (LMMs) for the solution of pure delay equations by making use of the three popular LMMs: Adams-Bashforth, AdamsMoulton and Backward Differentiation Formula.

Al-Mutib [3] also discussed the stability properties of numerical methods for DDEs by considering the onestep method, Runge-Kutta. In 1991, Hout and Spijker [4] analysed the stability of numerical methods for the solution of DDEs by studying the behaviour of these methods when they are applied to the linear test problem. Wille and Baker [5] considered the solution of DDEs by numerical methods and particularly, the numerical solution of state-dependent problems. Those worthy of mention in the discussion of the stability properties of numerical methods for DDEs include (but not limited to) Cryer [6], Barwell [7], Widerholt [8], Watanabe and Roth [9], Bellen and Zennaro [10], Brugnano and Trigiante [11]. Shakeri and Dehgan [12] employed the
Homotopy Perturbation Method (HPM) for the solution of DDEs. Some numerical illustrations were given in which they concluded that the proposed method is very efficient and easy to use.

FDEs are basically with constant delays, proportional delays, or time-dependent (time-varying) delays. Meanwhile, we will concentrate in this work on those types with proportional delays.

\section{Systems of delay differntial equations (SDDEs)}

Here, we give a brief introduction with respect to SDDEs as follows:

Let $f_{1}(\cdot), f_{2}(\cdot), f_{3}(\cdot), \cdots, f_{n}(\cdot) \quad$ be differentiable functions on a certain domain $D$, such that $q_{i} \in(0,1)$, $i=1(1) k$, and for some dimensional functions, $\underline{w}(t)=\left(w(t), w^{\prime}(t), w^{\prime \prime}(t), \ldots, w^{(n)}(t)\right)$, then:

$$
\left\{\begin{array}{c}
f_{l}\left(t, \underline{w}(t), \underline{w}\left(q_{0} t\right), \underline{w}\left(q_{l} t\right), \ldots, \underline{w}\left(q_{k} t\right)\right)=0 \\
f_{2}\left(t, \underline{w}(t), \underline{w}\left(q_{0} t\right), \underline{w}\left(q_{1} t\right), \ldots, \underline{w}\left(q_{k} t\right)\right)=0 \\
f_{3}\left(t, \underline{w}(t), \underline{w}\left(q_{0} t\right), \underline{w}\left(q_{1} t\right), \ldots, \underline{w}\left(q_{k} t\right)\right)=0 \\
\quad \vdots \\
f_{n}\left(t, \underline{w}(t), \underline{w}\left(q_{0} t\right), \underline{w}\left(q_{l} t\right), \ldots, \underline{w}\left(q_{k} t\right)\right)=0
\end{array}\right.
$$

is referred to as the general form of a SDDE with proportional delays, $q_{i} \in(0,1)$.

Equation (1) has vital applications in real life settings. Many researchers have applied series of methods such as HAM, ADM, and so on for solving various forms of differential equations including DDEs [13-15].

\footnotetext{
* Corresponding author: soedeki@yahoo.com
} 


\section{Analysis of the DTM}

The semi-analytical method (DTM) as remarked by many scholars, has been proven to be user-friendly in terms of application for differential models (linear or nonlinear), because the DTM converts the concerned problems to their equivalent forms in algebraic recursive relations, but this is not so when other semi-analytical techniques, such as VIM, HAM, ADM and so on are used [16-17]. The DTM has been modified to handle models of nonlinear types and the likes [18-21].

\subsection{The Overview of the DTM}

Let $m(t)$ be a differentiable function whose transformed form is $M(k)$. Thus, the $k$-th derivative of $m(t)$ is denoted as:

$$
M(k)=\frac{1}{k !}\left(\frac{d^{k} m(t)}{d t^{k}}\right)_{t=t_{0}} .
$$

So, the inverse form of $M(k)$ is:

$$
m(t)=\sum_{k=0}^{\infty} M(k)\left(t-t_{0}\right)^{k}
$$

Using (2) in (3) therefore gives:

$$
m(t)= \begin{cases}\sum_{k=0}^{\infty} \frac{\left(t-t_{0}\right)^{k}}{k !}\left(\frac{d^{k} m(t)}{d t^{k}}\right)_{t=t_{0}}, & t_{0} \neq 0 \\ \sum_{k=0}^{\infty} \frac{t^{k}}{k !}\left(\frac{d^{k} m(t)}{d t^{k}}\right)_{t=0}, & t_{0}=0\end{cases}
$$

Equation (4) signifies that the notion of the transformation method originated from Taylor series expansion.

\subsection{The fundamentals of DTM: theorems and properties}

Theorem 1: If $v(t)=\alpha v_{a}(t) \pm \beta v_{b}(t)$, then

$V(k)=\alpha V_{a}(k) \pm \beta V_{b}(k)$

Theorem 2: If $v(t)=\frac{\alpha d^{\eta} v_{+}(t)}{d t^{\eta}}, \eta \in \mathbb{N}$, then

$V(k)=\frac{\alpha(k+\eta) !}{k !} V_{+}(k+\eta)$

Theorem 3: If $v(t)=e^{\lambda t}$, then $V(k)=\frac{\lambda^{k}}{k !}$

Theorem 4: If $v(t)=v_{+}^{2}(t)$, then

$$
V(k)=\sum_{\eta=0}^{k} V_{+}(\eta) V_{+}(k-\eta)
$$

Theorem 5a: Suppose $h(t)$ and $v(t)$ are differentiable functions whose differential functions are $H(k)$ and $V(k)$ respectively and $p \in(0,1)$, then:

$H(k)=p^{k} V(k)$ if $h(t)=v(p t)$

where $p$ is the proportional delayed parameter.
Theorem 5b: If $v(t)=v_{1}\left(p_{1} t\right) v_{2}\left(p_{2} t\right)$, then

$$
V(k)=\sum_{\eta=0}^{k} p_{1}^{\eta} p_{2}^{k-\eta} V_{1}(\eta) V_{2}(k-\eta) \text {. }
$$

Note: the proof of theorems (1)-(4) can easily be found in standard texts [18], so we will briefly sketch that of theorem $5 \mathrm{a}$ as follows.

Proof: (Theorem 5a): Consider the derivative of $h(t)=v(p t)$ as follows for $\tau=(p t)$ and $h=h(t)$

That implies that:

$$
\begin{aligned}
& h=v(\tau), \frac{d h}{d \tau}=v^{\prime}(\tau) \text { and } d \tau=p d t\left(\text { i.e. } \frac{d \tau}{d t}=p\right) \\
& \therefore \quad \frac{d h}{d t}=\frac{d h}{d \tau} \cdot \frac{d \tau}{d t}=p \frac{d h}{d \tau}=p \frac{d v}{d \tau} .
\end{aligned}
$$

Similarly,

$$
\begin{aligned}
\frac{d^{2} h}{d t^{2}} & =\frac{d}{d t}\left(\frac{d h}{d t}\right)=\frac{d}{d t}\left(p \frac{d h}{d \tau}\right) \\
& =\frac{d}{d \tau}\left(p \frac{d h}{d \tau}\right) \frac{d \tau}{d t}=p^{2} \frac{d^{2} h}{d \tau^{2}}=p^{2} \frac{d^{2} v}{d \tau^{2}} .
\end{aligned}
$$

In general, the $k$-th derivative of $h(t)=v(p t)$ is:

$$
\frac{d^{k} h}{d t^{k}}=p^{k} \frac{d^{k} v}{d \tau^{k}}
$$

Hence, from (2)

$$
\begin{aligned}
& \left(\frac{d^{k} h}{d t^{k}}\right)_{t=t_{0}}=\left(p^{k} \frac{d^{k} v}{d \tau^{k}}\right)_{t=t_{0}}=p^{k}\left(\frac{d^{k} v}{d \tau^{k}}\right)_{t=t_{0}} \\
\therefore \quad\left(\frac{d^{k} h}{d t^{k}}\right)_{t=t_{0}} & =k ! p^{k} V(k) .
\end{aligned}
$$

So, dividing both sides of (9) by $k$ ! , and using (2) gives: $H(k)=p^{k} V(k)$

Q.E.D

\section{Illustrative examples and applications}

In this section, the proposed method is applied with some illustrative examples for the solutions of system of delay differential equations (SDDEs).

Case 1: Consider the following SDDE:

$$
\left\{\begin{array}{l}
h_{1}^{\prime}(t)=e^{-t}-e^{\frac{t}{2}}+h_{1}\left(\frac{t}{2}\right)+h_{1}(t)-h_{2}(t) \\
h_{2}^{\prime}(t)=e^{t}+e^{\frac{t}{2}}-h_{1}\left(\frac{t}{2}\right)-h_{1}(t)-h_{2}(t)
\end{array}\right.
$$

subject to:

$$
\left\{\begin{array}{l}
h_{1}(0)=1 \\
h_{2}(0)=1
\end{array}\right.
$$

with the exact solutions:

$$
\left\{\begin{array}{c}
h_{1}(t)=e^{t} \\
h_{2}(t)=e^{-t}
\end{array}\right.
$$


Solution procedure-Case 1:

Taking the DT of (10) gives:

$D T\left\{\begin{array}{l}h_{1}^{\prime}(t)=e^{-t}-e^{\frac{t}{2}}+h_{1}\left(\frac{t}{2}\right)+h_{1}(t)-h_{2}(t) \\ h_{2}^{\prime}(t)=e^{t}+e^{\frac{t}{2}}-h_{1}\left(\frac{t}{2}\right)-h_{1}(t)-h_{2}(t)\end{array}\right)$.

Therefore, for $k \geq 0$, we have the following in recurrence form:

$$
\begin{aligned}
H_{1}(k+1) & =\frac{1}{k+1}\left(\frac{(-1)^{k}}{k !}-\frac{\left(\frac{1}{2}\right)^{k}}{k !}\right. \\
\left.+\left(\frac{1}{2}\right)^{k} H_{1}(k)+H_{1}(k)-H_{2}(k)\right) & \\
H_{2}(k+1) & =\frac{1}{k+1}\left(\frac{(1)^{k}}{k !}+\frac{\left(\frac{1}{2}\right)^{k}}{k !}\right. \\
& \left.-\left(\frac{1}{2}\right)^{k} H_{1}(k)-H_{1}(k)-H_{2}(k)\right)
\end{aligned}
$$

with

$$
\left\{\begin{array}{l}
H_{1}(0)=1 \\
H_{2}(0)=1
\end{array} .\right.
$$

Thus, for $k=0$,

$$
\begin{gathered}
H_{1}(1)=\frac{1}{1}\left(\frac{(-1)^{0}}{0 !}-\frac{(0.5)^{0}}{0 !}\right. \\
\left.+\left(\frac{1}{2}\right)^{0} H_{1}(0)+H_{1}(0)-H_{2}(0)\right)=1 \\
H_{2}(1)=\frac{1}{1}\left(\frac{(1)^{0}}{0 !}+\frac{(0.5)^{0}}{0 !}\right. \\
\left.-\left(\frac{1}{2}\right)^{0} H_{1}(0)-H_{1}(0)-H_{2}(0)\right)=-1
\end{gathered}
$$

Thus, for $k=2$,

$$
\begin{aligned}
H_{1}(3) & =\frac{1}{3}\left(\frac{(-1)^{2}}{2 !}-\frac{(0.5)^{2}}{2 !}\right. \\
\left.+\left(\frac{1}{2}\right)^{2} H_{1}(2)+H_{1}(2)-H_{2}(2)\right)=\frac{1}{3 !} . & \\
H_{2}(3) & =\frac{1}{3}\left(\frac{(1)^{2}}{2 !}+\frac{(0.5)^{2}}{2 !}\right. \\
& \left.-\left(\frac{1}{2}\right)^{2} H_{1}(2)-H_{1}(2)-H_{2}(2)\right)=-\frac{1}{3 !} .
\end{aligned}
$$

Thus, for $k=3$,

$$
\begin{aligned}
H_{1}(4) & =\frac{1}{4}\left(\frac{(-1)^{3}}{3 !}-\frac{(0.5)^{3}}{3 !}\right. \\
\left.+\left(\frac{1}{2}\right)^{3} H_{1}(3)+H_{1}(3)-H_{2}(3)\right) & =\frac{1}{4 !} . \\
H_{2}(4) & =\frac{1}{4}\left(\frac{(1)^{3}}{3 !}+\frac{(0.5)^{3}}{3 !}\right. \\
& \left.-\left(\frac{1}{2}\right)^{3} H_{1}(3)-H_{1}(3)-H_{2}(3)\right)=\frac{1}{4 !} .
\end{aligned}
$$

Thus, for $k=4$,

$$
\begin{aligned}
H_{1}(5) & =\frac{1}{5}\left(\frac{(-1)^{4}}{4 !}-\frac{(0.5)^{4}}{4 !}\right. \\
& \left.+\left(\frac{1}{2}\right)^{4} H_{1}(4)+H_{1}(4)-H_{2}(4)\right)=\frac{1}{5 !} \\
H_{2}(5) & =\frac{1}{5}\left(\frac{(1)^{4}}{4 !}+\frac{(0.5)^{4}}{4 !}\right. \\
& \left.-\left(\frac{1}{2}\right)^{4} H_{1}(4)-H_{1}(4)-H_{2}(4)\right)=-\frac{1}{5 !} .
\end{aligned}
$$

Hence, considering (16)-(26), we write coefficients as follows:

$$
\begin{aligned}
& \left\{\begin{array}{l}
H_{1}(0)=1, H_{1}(1)=1, H_{1}(2)=\frac{1}{2 !}, \\
H_{1}(3)=\frac{1}{3 !}, H_{1}(4)=\frac{1}{4 !}, H_{1}(5)=\frac{1}{5 !}, \cdots
\end{array}\right. \\
& \left\{\begin{array}{l}
H_{2}(0)=1, H_{2}(1)=-1, H_{2}(2)=\frac{1}{2 !}, \\
H_{2}(3)=-\frac{1}{3 !}, H_{2}(4)=\frac{1}{4 !}, H_{2}(5)=-\frac{1}{5 !}, \cdots
\end{array}\right. \\
& \therefore h_{1}(t)=\sum_{k=0}^{\infty} H_{1}(k) t^{k}=H_{1}(0)+H_{1}(1) t+H_{1}(2) t^{2} \\
& =1+t+\frac{1}{2 !} t^{2}+\frac{1}{3 !} t^{3}+\frac{1}{4 !} t^{4}+\frac{1}{5 !} t^{5}+\cdots
\end{aligned}
$$

Similarly, 


$$
\begin{gathered}
h_{2}(t)=\sum_{k=0}^{\infty} H_{2}(k) t^{k}=H_{2}(0)+H_{2}(1) t+H_{2}(2) t^{2} \\
+H_{2}(3) t^{3}+H_{2}(4) t^{4}+\cdots \\
=1-t+\frac{1}{2 !} t^{2}-\frac{1}{3 !} t^{3}+\frac{1}{4 !} t^{4}-\frac{1}{5 !} t^{5}+\cdots .
\end{gathered}
$$

Case 2: Consider the SDDE [17]:

$$
\left\{\begin{array}{l}
2 h_{1}^{\prime}(t)=h_{1}(t)+e^{\frac{t}{2}} h_{2}\left(\frac{t}{2}\right) \\
2 h_{2}^{\prime}(t)=h_{2}(t)+e^{\frac{t}{2}} h_{1}\left(\frac{t}{2}\right)
\end{array}\right.
$$

subject to:

$$
h_{1}(0)=1=h_{2}(0)
$$

with the exact solutions:

$$
\left\{\begin{array}{l}
h_{1}(t)=e^{t} \\
h_{2}(t)=e^{t}
\end{array} .\right.
$$

Solution procedure-case 2 :

Taking the DT of (30) gives:

$$
D T\left(\left\{\begin{array}{l}
2 h_{1}^{\prime}(t)=h_{1}(t)+e^{\frac{t}{2}} h_{2}\left(\frac{t}{2}\right) \\
2 h_{2}^{\prime}(t)=h_{2}(t)+e^{\frac{t}{2}} h_{1}\left(\frac{t}{2}\right)
\end{array}\right) .\right.
$$

Therefore, for $k \geq 0$, we have the following in recurrence form:

$$
\begin{aligned}
& H_{1}(k+1)=\frac{1}{2(k+1)} \\
& \left(H_{1}(k)+\sum_{i=0}^{k} \frac{(0.5)^{i}}{i !}\left(\frac{1}{2}\right)^{k-i} H_{2}(k-i)\right), \\
& H_{2}(k+1)=\frac{1}{2(k+1)} \\
& \left(H_{2}(k)+\sum_{i=0}^{k} \frac{(0.5)^{i}}{i !}\left(\frac{1}{2}\right)^{k-i} H_{1}(k-i)\right),
\end{aligned}
$$

with

$$
\left\{\begin{array}{l}
H_{1}(0)=1 \\
H_{2}(0)=1
\end{array} .\right.
$$

Hence, using (36) in (34)-(35) for $k \in \mathbb{N} \cup\{0\}$, we have:

$$
\begin{aligned}
& \left\{\begin{array}{l}
H_{1}(1)=1, H_{1}(2)=\frac{1}{2}, H_{1}(3)=\frac{1}{6}, H_{1}(4)=\frac{1}{24}, \\
H_{1}(5)=\frac{1}{120}, \cdots
\end{array}\right. \\
& \left\{\begin{array}{l}
H_{2}(1)=1, H_{2}(2)=\frac{1}{2}, H_{2}(3)=\frac{1}{6}, H_{2}(4)=\frac{1}{24}, \\
H_{2}(5)=\frac{1}{120}, \cdots
\end{array}\right.
\end{aligned}
$$

$$
\begin{aligned}
\therefore h_{1}(t)=\sum_{k=0}^{\infty} H_{1}(k) t^{k} & =H_{1}(0)+H_{1}(1) t+H_{1}(2) t^{2} \\
& +H_{1}(3) t^{3}+H_{1}(4) t^{4}+\cdots \\
= & 1+t+\frac{1}{2} t^{2}+\frac{1}{6} t^{3}+\frac{1}{24} t^{4}+\frac{1}{120} t^{5}+\cdots \approx e^{t} .
\end{aligned}
$$

Similarly,

$$
\begin{gathered}
h_{2}(t)=\sum_{k=0}^{\infty} H_{2}(k) t^{k}=H_{2}(0)+H_{2}(1) t+H_{2}(2) t^{2} \\
+H_{2}(3) t^{3}+H_{2}(4) t^{4}+\cdots \\
=1+t+\frac{1}{2} t^{2}+\frac{1}{6} t^{3}+\frac{1}{24} t^{4}+\frac{1}{120} t^{5}+\cdots \approx e^{t} .
\end{gathered}
$$

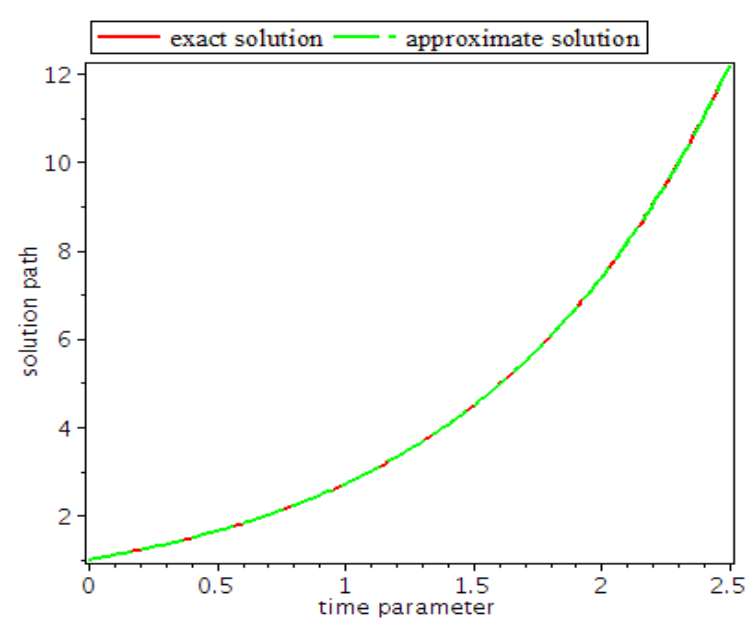

Fig. 1: Exact and Approx. Solutions of $h_{1}(t)$ in case 1

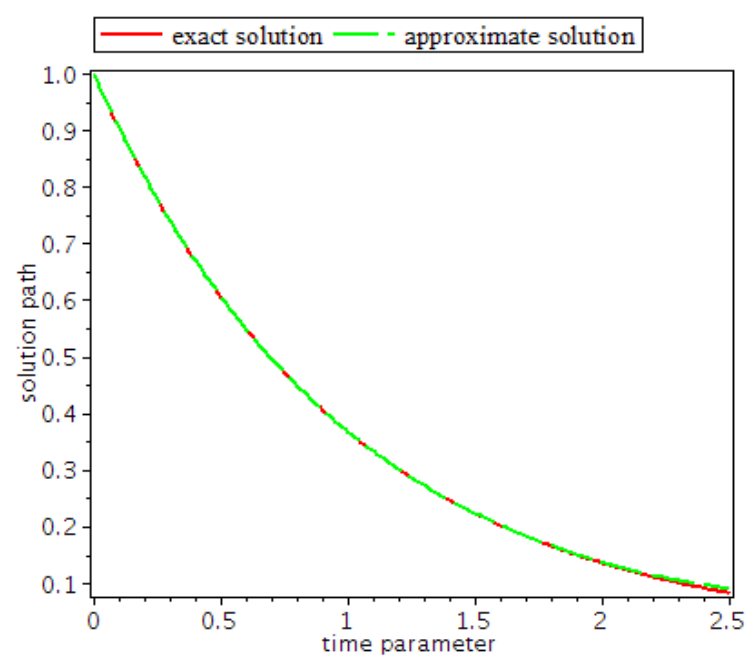

Fig. 2: Exact and Approx. Solution of $h_{2}(t)$ in case 1 


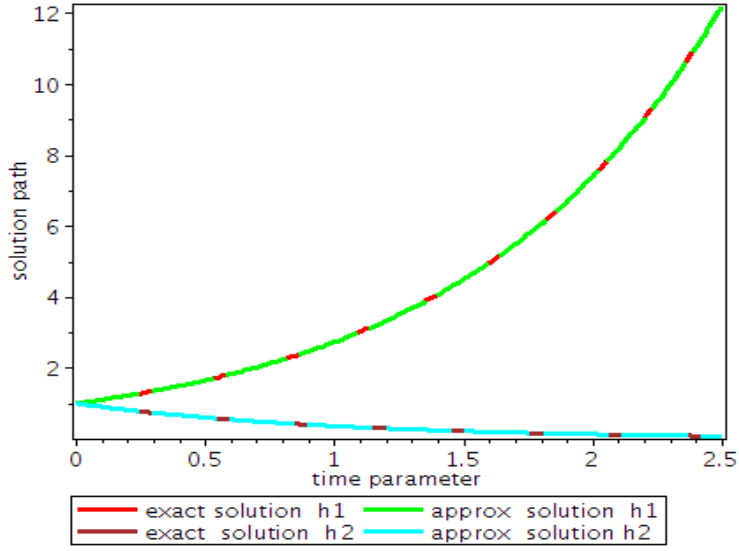

Fig. 3: Exact and Approx. Solutions of $h_{1}(t) \& h_{2}(t)$ in case 1

\section{CONCLUSION}

We considered in this work, by means of Zhou method, the approximate-analytical solutions of a certain system of functional differential equations (SFDEs) engendered by proportional delay. The effectiveness and robustness of the proposed technique was illustratively demonstrated and the results coincide approximately with their exact forms when compared. In addition, we noted that using this method, the SFDEs with proportional delays need not be converted to SFDEs with constant delays before obtaining their solutions, and no symbolic calculation or initial guesstimates, linearization or perturbation is required.

\section{Acknowledgement}

The authors are grateful to Covenant University for financial support. In addition, they also wish to thank the anonymous reviewer(s) for their constructive comments.

\section{References}

[1] J. C. Butcher, "Numerical Methods for Ordinary Differential Equations". New York: John Wiley \& Sons (2008).

[2] P. J. Van der Houwen, and B. P. Sommeijer, "Stability in Linear Multistep Methods for Pure Delay Equations", J. Comput. Appl. Math., 10, (1984): 55-63.

[3] A. N. Al-Mutib, "Stability Properties of Numerical Methods for Solving Delay Differential Equations", J. Comput. Appl. Math., 10, (1984): 71-79.

[4] K. J. in't Hout and M. N. Spijker, "Stability Analysis of Numerical Methods for Delay Differential Equations", Numer. Math., 59, (1991): 807-814.

[5] D. R. Wille, and C. T. H. Baker, "Stepsize Control and Continuity Consistency for State-Dependent DelayDifferential Equations", J. Comput. Appl. Math., 53, (1994): 163-170.
[6] C.W. Cryer, "Highly-Stable Multistep Methods for Retarded Differential Equations", SIAM J. Numer. Anal. 11, (1974): 788-797.

[7] V. K. Barwell, "Special Stability Problems for Functional Differential Equations”, BIT, 15, (1975): 130-135.

[8] L. F. Widerholt, "Stability of Multistep Methods for Delay Differential Equations", Math. Comput., 30 (134), (1976): 283-290.

[9] D. S. Watanabe, and M. Roth, "The Stability of Difference Formulas for Delay Differential Equations", SIAM J. Numer. Anal., 22, (1985): 132-145.

[10] A. Bellen, and M. Zennaro, "Strong Contractivity Properties of Numerical Methods for Ordinary and Delay Differential Equations", Appl. Numer. Math., 9, (1992): 321-346.

[11] L. Brugnano and D. Trigiante, "Solving Differential Problems by Multistep Initial and Boundary Value Methods". Amsterdam: Gordon and Breach Science Publishers (1998).

[12] F. Shakeri, and M. Dehghan, "Solution of delay differential equations via a homotopy perturbation method", Mathematical and Computer Modelling, 48, (2008): 486-498.

[13] M. Zennaro, "Delay Differential Equations: Theory and Numerics, in Theory and Numerics of Ordinary and Partial Differential Equations", Adv. Numer. Anal., 5, Clarendon Press, Oxford, (1995).

[14] G.O. Akinlabi and S. O. Edeki, "On Approximate and Closed-form Solution Method for Initial-value Wave-like Models", International Journal of Pure and Applied Mathematics, 107 (2), (2016): 449-456.

[15] L. Qiu, T. Mitsui, and J.-X. Kuang, "The numerical stability of the $\theta$-method for delay differential equations with many variable delays", Journal of Computational Mathematics, 17 (5) (1999): 523-532.

[16] S.O. Edeki, G.O. Akinlabi and S.A. Adeosun, "Analytic and Numerical Solutions of Time-Fractional Linear Schrödinger Equation", Communications in Mathematics and Applications, 7 (1), (2016): 1-10.

[17] R. K. Saeed and B. M. Rahman, "Adomian Decomposition Method for Solving System of Delay Differential Equation", Australian Journal of Basic and Applied Sciences, 4 (8) (2010): 3613-3621.

[18] J. K. Zhou, "Differential Transformation and its Application in Electrical Circuits", Huazhong University Press, Wuhan, China, (1986).

[19] S. O. Edeki, G. O. Akinlabi, S. A. Adeosun, "On a modified transformation method for exact and approximate solutions of linear Schrödinger equations", AIP Conference proceedings 1705, 020048 (2016); doi: 10. $1063 / 1.4940296$.

[20] Gh. J. Mohammed, F. S. Fadhel, "Extend differential transform methods for solving differential equations with multiple delay", Ibn Al-Haitham J. for Pure and Appl. Sci., 24 (3), (2011): 1-5.

[21] S. O. Edeki, O. O. Ugbebor, E. A. Owoloko, "Analytical Solutions of the Black-Scholes Pricing Model for European Option Valuation via a Projected Differential Transformation Method", Entropy, 17 (11), (2015): 75107521.

[22] J. Rebenda, Z. Smarda, Y. Khan, "A Taylor Method Approach for Solving of Nonlinear Systems of Functional Differential Equations with Delay", arXiv:1506.05646v1, [math.CA], 18 June, (2015). 